\title{
Thermal origin of neutron star magnetic fields
}

\author{
R. D. Blandford, J. H. Applegate and L. Hernquist \\ 130-33 Caltech, Pasadena, California 91125, U.S.A.
}

Received 1982 December 7; in original form 1982 September 22

Summary. It is proposed that magnetic field arises naturally in neutron stars as a consequence of thermal effects occurring in their outer crusts. The heat flux through the crust, which is carried mainly by degenerate electrons, can give rise to a possible thermoelectric instability in the solid crust which causes horizontal magnetic field components to grow exponentially with time. However, in order for the thermally driven growth to exceed ohmic decay, either the electron collision time must exceed existing estimates by a factor $\sim 3$ or the surface layers comprise helium. A second instability is possible if the liquid phase that lies above the solid crust also contains a horizontal magnetic field. The heat flux will drive circulation which should amplify the field strength provided that there is a seed field in excess of $\sim 10^{8} \mathrm{G}$.

If either of these two instabilities develops the field will quickly grow to a strength of $\sim 10^{12} \mathrm{G}$, where the instabilities become non-linear. Further growth will saturate when either the magnetic stress exceeds the lattice yield stress or the temperature perturbations become non-linear, both of which occur at a subsurface field strength of $\sim 10^{14} \mathrm{G}$; the corresponding surface field strength is $\sim 10^{12} \mathrm{G}$. Further evolution of the magnetic field should lead to long-range order and yield neutron star magnetic dipole moments $\sim 10^{30} \mathrm{G} \mathrm{cm}^{3}$, comparable with those observed.

Newly-formed neutron stars should be able to develop their dipole moments in a hundred thousand years and maintain them for as long as heat flows through the crust. Thereafter, the dipole moment should decay in several million years, as observed in the case of most radio pulsars. Neutron stars that are formed spinning rapidly, like that in the Crab Nebula, should be able to grow magnetic fields far more rapidly since their rotational energy can also be tapped to drive thermoelectric currents. The interiors of neutron stars in binary systems may be heated by the energy released by accreting matter. The resulting heat flux may cause the production of magnetic fields in these objects. Binary pulsars, with their unusually low and persistent fields, have probably passed through this phase. 


\section{Introduction}

Timing observations indicate that surface magnetic fields of radio pulsars $\left[\propto(P \dot{P})^{1 / 2}\right.$, where $P$ is the period and $\dot{P}$ is the period derivative] have values in the range $1-5 \times 10^{12} \mathrm{G}$, with surprisingly little scatter (e.g. Manchester 1981). This range of neutron star surface field strengths has been corroborated by the direct observation of cyclotron lines in Her X-1 (Trümper et al. 1978), 4U $0115+63$ (Wheaton et al. 1979), GX1 + 4 (Maurer et al. 1982), 4U 1626 - 67 (Pravdo et al. 1979) and the Crab Pulsar (Manchanda et al. 1982). Analyses of period changes in pulsating X-ray sources (e.g. Rappaport \& Joss 1977; Ghosh \& Lamb 1979) caused by magnetic coupling between the neutron star and the accreting plasma yield values of neutron star magnetic moments consistent with surface fields $\sim 10^{12} \mathrm{G}$. The spectra of gamma-ray bursts show features interpretable as cyclotron absorption lines, as well as possible positron annihilation features, indicating that the burst sources are magnetized neutron stars with surface fields $\sim 10^{12} \mathrm{G}$ (Mazets et al. 1981; but see Fenimore et al. 1982).

There are indications that the magnetization of neutron stars is not permanent. Radio pulsars have a scale height $\sim 500 \mathrm{pc}$ above the galactic plane and one-dimensional velocities $\sim 100 \mathrm{~km} \mathrm{~s}^{-1}$ away from the galactic plane, strongly suggesting that they are active for no more than a few million years (e.g. Lyne 1981). Comparison of the kinetic ages (distance above the galactic plane/velocity normal to the galactic plane) of pulsars with the timing ages, $P / 2 \dot{P}$, shows serious disagreement. The kinetic age, believed to be the true age, is shorter than the timing age for pulsars older than a few million years (e.g. Lyne 1981), a fact that may be concluded independently from the joint distribution of pulsars in $P$ and $\dot{P}$ (e.g. Lyne 1981). One long-standing explanation for these difficulties (e.g. Gunn \& Ostriker 1970 ) is that the magnetic field decays in a few million years, suppressing coherent radio emission and increasing the timing age (alternative explanations may be found in Goldreich 1970; Flowers \& Ruderman 1977; Vivekanand \& Radhakrishnan 1981). Further evidence for a changing magnetization is supplied by galactic X-ray sources, the majority of which show no evidence of pulsation and presumably involve emission from an entire neutron star surface, rather than just a polar cap (e.g. Joss 1980; but see Inoue et al. 1981).

The simplest mechanism for field decay is ohmic diffusion; however, apart from the outermost crust, neutron stars cannot ohmically dissipate a magnetic field in a million years. The protons in the core of a neutron star are believed to form a type II superconductor; any flux threading the core when the star cools below the transition temperature will be trapped there, essentially forever. At densities below nuclear, where there are no superconducting protons, the electrical conductivity is far too high to allow ohmic dissipation in a million years. Thus, if field decay is to occur, it appears essential that the surface flux penetrate only to a depth $\lesssim 1 \mathrm{~km}$, where the electrical conductivity is low enough to allow ohmic dissipation to operate on the requisite time-scale. The subsurface field would then be $\gtrsim 10^{13} \mathrm{G}$, assuming $10^{12} \mathrm{G}$ fields at the polar caps. A field of this size will cause a substantial modification of the surface structure and transport properties.

There is an additional reason for believing that neutron stars possess subsurface horizontal fields. In the pulsating X-ray sources, matter is believed to be accreted at the magnetic poles of the neutron star. However, there must be a continuous flow of matter from the poles to the equatorial regions in order for the star to re-establish hydrostatic equilibrium. If the stellar field were just a simple dipole, the matter would sink to a depth at which its pressure could overcome the magnetic stresses. At this point the matter would spread sideways, dragging the magnetic field lines with it, as ohmic dissipation is quite ineffective on a flow time-scale. (Blandford, DeCampli \& Königl 1979).

There is evidence that neutron stars become magnetized after they are formed. Observations of the supernova remnant MSH 15-52 and its embedded pulsar (Seward \& Harnden 
1982; Seward et al. 1982; Weisskopf et al. 1982) show a pulsar with a timing age of $P / 2 \dot{P}=$ $1550 \mathrm{yr}$ in a supernova remnant estimated to be $\sim 10^{4} \mathrm{yr}$ old. The apparent ages, discrepant if the pulsar field is a stellar fossil, are reconciled if the neutron star is as old as the SNR, but became a pulsar $\sim 10^{3} \mathrm{yr}$ ago when its magnetic field grew to sufficient strength. Vivekanand $\&$ Narayan (1981) have suggested on the basis of the distribution of pulsars in $P, \dot{P}$ that the majority of neturon stars do not become pulsars until long after being formed. Observations of the supernova remnant RCW 103 (Tuohy \& Garmire 1980) show a hot neutron star but no radio pulsar or 'plerionic' nebula, suggesting that the neutron star does not possess a magnetic field. The X-ray source Her X-1 is estimated to be $\sim 10^{8} \mathrm{yr}$ old (van den Heuvel 1977) and yet possesses a magnetic field of $5 \times 10^{12} \mathrm{G}$ (Trümper et al. 1978). The unusual longevity of the field, difficult to understand if the field is a fossil, is explained if the field is a product of the accretion process that powers the X-ray source.

Detailed cooling calculations (e.g. Tsuruta 1979; Glen \& Sutherland 1980; Nomoto \& Tsuruta 1981; Van Riper \& Lamb 1981; Richardson et al. 1982) have shown that the interior temperature of a neutron star falls from $>10^{9} \mathrm{~K}$ to $\sim 10^{8} \mathrm{~K}$ in the first $10^{5} \mathrm{yr}$ of its life. At these early times the neutron star cools predominantly by neutrino emission; however, there is still a heat flux through the crust, decreasing from $\sim 10^{22}$ to $\sim 10^{19} \mathrm{erg} / \mathrm{cm}^{2} \mathrm{~s}$, as the star cools. This heat flux, insignificant in the total energetics, is quite important observationally; it is the source of the thermal X-rays observed from neutron stars (e.g. Helfand, Chanan \& Novick 1980). At densities above $10^{5} \mathrm{gm} / \mathrm{cm}^{3}$ the heat transport is by electron conduction; at lower densities photon transport dominates. The magnitude of the heat flux is largely determined by the electron conduction opacity in the liquid $\left(10^{6} \mathrm{gm} / \mathrm{cm}^{3} \leqslant\right.$ $\rho \leqslant 10^{8} \mathrm{gm} / \mathrm{cm}^{3}$ for $T \sim 10^{8} \mathrm{~K}$ ) (Gudmundsson, Pethick \& Epstein 1982).

The principal effect leading to field generation can be understood as follows. Suppose that there is a small horizontal component of magnetic field of strength $B$. Hot electrons from below will be deflected horizontally by the field; cooler electrons from above will be deflected slightly less in the opposite direction. The net effect is to produce a horizontal heat flux, $F_{\perp} \sim(e \tau / \mu) \vec{B} \times \vec{F}$, where $e=|e|$ is the magnitude of the electronic charge, $\tau$ is the electron collision time, $\mu$ is the electron chemical potential and $\vec{F}$ is the vertical heat flux (we use units in which $c=k_{\mathrm{B}}=\hbar=1$ ). Fourier components of the magnetic field with horizontal wavelength comparable with the depth $z$ create horizontal temperature gradients $\sim F_{\perp} / \kappa$, where $\kappa$ is the thermal conductivity. The pressure of a degenerate, relativistic free electron gas is $P\left(n_{\mathrm{e}}, T\right)=P\left(n_{\mathrm{e}}, T=0\right)+\left(\pi^{2} / 6\right) n_{\mathrm{e}} T^{2} / \mu$. Hence there is an additional pressure gradient $\sim n_{\mathrm{c}} T \nabla T / \mu$, which must be balanced by a thermoelectric field $E \sim T \nabla T / \mu e \sim$ $B F / \mu n_{\mathrm{e}}$. This field has a non-vanishing curl and so $-\overrightarrow{\mathrm{V}} \times \vec{E}=\partial \vec{B} / \partial t=\Gamma_{+} \vec{B}$, where $\Gamma_{+} \sim F / \mu n_{\mathrm{e}} z$. The growth rate $\Gamma_{+}$is positive when the heat flows down the density gradient. If we substitue characteristic values $F=10^{20} \mathrm{erg} / \mathrm{cm}^{2} \mathrm{~s}, \mu=4 \mathrm{MeV}, n_{\mathrm{e}}=3 \times 10^{31} \mathrm{~cm}^{-3}, z=50 \mathrm{~m}$ then $\Gamma_{+} \sim$ $300 \mathrm{yr}^{-1}$. It is clear from this estimate, which does not depend directly upon the local transport coefficients, that there may be time for the field to grow as the star cools. Note that the potential difference across the crust associated with this electric field is only $\sim\left(B / 10^{8} \mathrm{G}\right) \mathrm{mV}$, far smaller than the potential differences induced by gravity and rotation. The important point is that this field alone has a non-vanishing curl.

It is necessary for the growth rate $\Gamma_{+}$to exceed the ohmic decay rate $\Gamma_{-}$if the field is to grow. This may be estimated by $\Gamma_{-} \sim 1 / 4 \pi \sigma z^{2}$. Substituting $\sigma \sim 10^{21} \mathrm{~s}^{-1}$ appropriate to these conditions yields a decay rate comparable to the growth rate. A more detailed calculation is necessary to determine if neutron star magnetic fields can have a thermal origin.

The idea of using thermoelectric currents to generate astrophysical fields is not new. Biermann (1950) (cf. Mestel 1961; Roxburgh 1966) showed that differential rotation in fluid stars can produce a misalignment of the isobars and the equipotentials, resulting in a 
battery effect. This idea, and its terrestrial counterpart (e.g. Hibberd 1979), have generally not found favour because the effect is quantitatively quite small and dynamo action induced by helical motion in the fluid is thought to be far more effective (e.g. Parker 1979; and in the neutron star case see Ruderman \& Sutherland 1973). Thermoelectric effects can be far more important in a neutron star because standard dynamo action is inoperative in the solid crust.

- This mechanism does have a laboratory counterpart. Megagauss magnetic fields are routinely produced in the coronal plasma surrounding laser fusion targets; however, the details of the mechanism are rather different because inertial effects allow a departure from hydrostatic equilibrium in this case (e.g. Stamper et al. 1971).

Linear growth of the field is impossible in the liquid phase. However, when the field exceeds $\sim 10^{8} \mathrm{G}$, magnetic perturbations to the heat flow will drive circulatory motion in the fluid with turnover times that are short compared with the ohmic decay time. These motions will probably induce dynamo-like action and can further enhance the field strength at the solid surface.

In the following section we summarize and extend the analysis of thermoelectric phenomena in degenerate stars due to Urpin \& Yakovlev (1980b) and calculate necessary conditions for field generation. In Section 3, a linearized calculation of the growth of the field in the solid crust is given, which demonstrates that small seed fields can grow exponentially for sufficiently large heat fluxes. Non-linear growth of the field in the liquid is described in Section 4 . If the field strength can grow to $\sim 10^{11} \mathrm{G}$, the electron gyrofrequency will exceed the collision frequency and the field growth in the solid will enter the non-linear phase. The Hall effect will lead to rapid convection of magnetic flux and the creation of progressively larger scale structure, perhaps resulting in the establishment of an axisymmetric field geometry. In the absence of external heat sources, the interior of the star will cool and the field will decay. These issues are discussed in Section 5. In Section 6 we outline some of the observational consequences of this theory for pulsars, binary X-ray sources, X-and $\gamma$-ray bursters and white dwarfs.

\section{Thermoelectric effects}

We are concerned with the properties of the outer crust in the density range $10^{7} \mathrm{gm} / \mathrm{cm}^{3} \leqslant$ $\rho \leqslant 10^{11} \mathrm{gm} / \mathrm{cm}^{3}$, and for temperatures $T \sim 10^{8} \mathrm{~K}$. Under these conditions the electron gas is degenerate, and may be treated as ultrarelativistic and free to an accuracy of better than a few per cent. In the presence of electric fields and gradients of chemical potential and temperature the laws of charge and heat transport are

$\vec{j}=\overrightarrow{\vec{\sigma}} \cdot \vec{\epsilon}-\overrightarrow{\bar{\lambda}} \cdot \vec{\nabla} T$

and,

$\vec{F}=T \overrightarrow{\vec{\lambda}} \cdot \vec{\epsilon}-\overrightarrow{\vec{\gamma}} \cdot \vec{\nabla} T$

where the electrochemical field $\vec{\epsilon}$ is the sum of the electric field and the chemical potential gradient $\vec{\epsilon}=\vec{E}+\vec{\nabla} \mu / e$ (cf. Landau \& Lifshitz 1960; Ashcroft \& Mermin 1976; Ziman 1972). In these expressions $\vec{j}$ is the electrical current, $\vec{F}$ is the heat current and $\vec{\sigma}$ is the electrical conductivity. The thermal conductivity $\overrightarrow{\vec{k}}$ and thermopower $\vec{Q}$ are related to the coefficients $\overrightarrow{\vec{\lambda}}$ and $\vec{\gamma}$ by

$\overrightarrow{\dot{Q}}=(\overrightarrow{\vec{\sigma}})^{-1} \cdot \overrightarrow{\vec{\lambda}}$

$\overrightarrow{\bar{k}}=\overrightarrow{\vec{\gamma}}-T \overrightarrow{\bar{Q}} \cdot \overrightarrow{\bar{\lambda}}$ 
The difference between $\overrightarrow{\vec{k}}$ and $\vec{\gamma}$ is of order $(T / \mu)^{2}$ for a free electron gas and will be neglected in this paper. Various thermogalvanomagnetic effects (Hall, Nernst, Leduc-Righi and Ettingshausen; $c f$. Landau \& Lifshitz 1960) result from electric currents driven by temperature gradients, heat currents driven by electric fields, and the magnetic field dependence of the coefficients in equation $(2.1 \mathrm{a}, \mathrm{b})$. The magnetization of the crust is much smaller than the magnetic field (Blandford \& Hernquist 1982) and will be neglected.

Expressions for the transport coefficents $\overrightarrow{\vec{\sigma}}, \overrightarrow{\vec{k}}$ and $\overrightarrow{\vec{\lambda}}$ are derived from the relativistic Boltzmann transport equation

$\vec{v} \cdot \vec{\nabla}_{\mathrm{r}} f-e(\vec{E}+\vec{v} \times \vec{B}) \cdot \vec{\nabla}_{\mathrm{k}} f=\int \frac{d^{3} k^{\prime}}{(2 \pi)^{3}} W\left(\vec{k}, \vec{k}^{\prime}\right)\left[f(\vec{r}, \vec{k})-f\left(\vec{r}, \vec{k}^{\prime}\right)\right]$

where $f(\vec{r}, \vec{k})$ is the electron distribution function and $W(\vec{k}, \vec{k})$ is the scattering rate. The scattering of electrons is by individual ions in the case of a liquid, and by phonons in the case of a solid. The scattering in the liquid is always elastic (ion mass $\gg$ electron mass); the scattering by phonons is elastic if the temperature exceeds the Debye temperature. As these are the important scattering mechanisms for the case of interest, elastic scattering is assumed and the energy-dependent relaxation time approximation used ( $c f$. Yakovlev \& Urpin 1980).

The Boltzmann equation (2.3), is solved by linearization in the standard manner ( $c f$. Ashcroft \& Mermin 1976). The distribution function is split into a Fermi-Dirac distribution, $f^{(0)}$, evaluated at the local values of $T$ and $\mu$ and a perturbation $\delta f$ responsible for transporting heat, charge, etc. The scattering term in (2.3) is replaced by $-\delta f / \tau$, where the relaxation time $\tau$ is given by

$\tau\left(\epsilon_{\mathrm{k}}\right)^{-1}=\int \frac{d^{3} k^{\prime}}{(2 \pi)^{3}} W\left(\epsilon_{\mathrm{k}}, \hat{k} \cdot \hat{k}^{\prime}\right)\left[1-\hat{k} \cdot \hat{k}^{\prime}\right]$

where $\epsilon_{\mathrm{k}}=\left(k^{2}+m^{2}\right)^{1 / 2}$ is the single particle energy. The resulting momentum space differential equation is integrated along unperturbed orbits to give

$\delta f=\left(-\frac{\partial f^{(0)}}{\partial \epsilon_{\mathrm{k}}}\right) \tau \vec{v} \cdot \overrightarrow{\bar{\chi}} \cdot\left[e \vec{E}+\vec{\nabla} \mu+\left(\frac{\epsilon_{\mathrm{k}}-\mu}{T}\right) \nabla T\right]$

where the inverse of the tensor $\overrightarrow{\bar{\chi}}$ is

$\left(\chi^{-1}\right)_{\mathrm{ij}}=\delta_{\mathrm{ij}}+\epsilon_{\mathrm{ijk}} X_{\mathrm{k}}$

with $\vec{X}=(e \tau / \mu) \vec{B}$.

Expressions for the transport coefficients $\vec{\sigma}, \vec{\kappa}$ and $\overrightarrow{\bar{\lambda}}$ are obtained by substitution of (2.5) into expressions for the currents

$\vec{j}=-2 e \int \frac{d^{3} k}{(2 \pi)^{3}} \vec{v} \delta f$

$\vec{F}=2 \int \frac{d^{3} \mathrm{k}}{(2 \pi)^{3}} \vec{v}\left(\epsilon_{\mathrm{k}}-\mu\right) \delta f$.

The electrical current, $(2.7 \mathrm{a})$ is the current of electrons times the charge per electron. The thermal current, $(2.7 \mathrm{~b})$, is obtained by applying the first law of thermodynamics to a fixed volume. The change in energy is $-\vec{\nabla} \cdot \int\left(\epsilon_{\mathrm{k}}+\phi\right) \vec{v} \delta f$ where $\phi$ is the single particle potential. 
However, adding particles with energy equal to the chemical potential adds no heat. To obtain the heat added $-\vec{\nabla} \cdot(\mu+\phi) \int \vec{v} \delta f$ must be subtracted from the energy added, where $\mu$ is the chemical potential of the system in the absence of the single particle potential. Writing the heat added as the negative of a divergence gives $(2.7 \mathrm{~b})$ for the heat current. The transport coefficients obtained by substitution of (2.5) into $(2.7 \mathrm{a}, \mathrm{b})$ are

$$
\begin{aligned}
& \overrightarrow{\vec{\sigma}}=\sigma_{0} \overrightarrow{\bar{\chi}}=\frac{n_{\mathrm{e}} e^{2} \tau}{\mu} \overrightarrow{\bar{\chi}}, \\
& \overrightarrow{\bar{\kappa}}=\kappa_{0} \overrightarrow{\bar{\chi}}=\frac{\pi^{2} n_{\mathrm{e}} T \tau}{3 \mu} \overrightarrow{\bar{\chi}}
\end{aligned}
$$

and

$$
\overrightarrow{\vec{\lambda}}=-\frac{\pi^{2} T}{3 e} \frac{d \vec{\sigma}}{d \mu}
$$

where $n_{\mathrm{e}}$ is the density of electrons.

Equation (2.1 a) can be written in the form of a fluid equation as follows. Use the thermodynamic identity $d \mu=d P / n_{\mathrm{e}}-S_{\mathrm{e}} d T$, where $S_{\mathrm{e}}=\pi^{2} T / p_{\mathrm{F}} v_{\mathrm{F}}$ is the entropy per electron of the electrons, and multiply equation (2.1a) by the inverse of the electrical conductivity tensor, (2.8), to obtain

$$
-e n_{\mathrm{e}} \vec{E}+\vec{j} \times \vec{B}-\vec{\nabla} P_{\mathrm{e}}+\frac{\mu \vec{j}}{e \tau}-\vec{F} \frac{d(\mu / \tau)}{d \mu}=0
$$

where $-\overrightarrow{\vec{K}} \cdot \vec{\nabla} T$ has been replaced by the heat flux $\vec{F}$, which is accurate to order $(T / \mu)^{2}$. The direct influence of gravity upon the electrons has been neglected, which is acceptable for $\mu \ll m_{\mathrm{p}}$.

The first two terms in (2.11) constitute the Lorentz force per unit volume acting upon the electron gas. The third term is the electron pressure gradient. The fourth and fifth terms are derived from the force density exerted by the lattice upon the electrons, which may be written as

$$
-2 \int \frac{d^{3} k}{(2 \pi)^{3}} \frac{\vec{p}}{\tau} \delta f=\frac{\mu \vec{j}}{e \tau}-\vec{F} \frac{d(\mu / \tau)}{d \mu}
$$

expanding in a Taylor series about $\epsilon_{\mathrm{k}}=\mu$. In a non-relativistic system with an energy independent relaxation time the vanishing of the electrical current implies the vanishing of the force density (2.12). This is not the case in a relativistic system because the ratio of momentum to velocity, $k / v=\epsilon_{\mathrm{k}}$, is variable; thus, the second term in (2.12) can contribute even if the first term vanishes and $\tau$ is constant.

The equation of hydrostatic equilibrium for the crust as a whole is

$-\vec{\nabla} P_{\mathrm{e}}-\rho \vec{\nabla} \phi+\vec{j} \times \vec{B}+\vec{Y}=0$

where $\phi$ is the gravitational potential, including centrifugal terms, and $\vec{Y}$ is the force density given by the divergence of the lattice stress tensor. The total charge density is negligible on length scales much larger than the Debye length. In writing the centrifugal terms as the gradient of a potential we have assumed that the crust is not differentially rotating. The equation of hydrostatic balance for the ionic lattice is the difference of (2.13) and (2.11)

$e n_{\mathrm{e}} \vec{E}-\rho \vec{\nabla} \phi-\frac{\overrightarrow{\mu j}}{e \tau}+\vec{Y}+\vec{F} \frac{d(\mu / \tau)}{d \mu}=0$. 
To calculate the growth rate of the field write (2.1a) as $\vec{\epsilon}=\overrightarrow{\vec{\sigma}}^{-1} \cdot \vec{j}+\vec{Q} \cdot \vec{\nabla} T$, and take the curl, using Faraday's law to obtain

$\frac{\partial \vec{B}}{\partial t}=-\vec{\nabla} \times \vec{\epsilon}=\vec{\nabla} \times(\vec{V} \times \vec{B})-\vec{\nabla} Q_{0} \times \vec{\nabla} T-\vec{\nabla} \times\left[\frac{\vec{\nabla} \times \vec{B}}{4 \pi \sigma_{0}}\right]$

with

$\vec{V}=\frac{\overrightarrow{\tilde{k}} \cdot \vec{\nabla} T}{n_{\mathrm{e}} \mu} \frac{d \ln (\mu / \tau)}{d \ln \mu}-\frac{\vec{j}}{e n_{\mathrm{e}}}$

and where

$Q_{0}=-\frac{\pi^{2} T}{3 e \mu} \frac{d \ln \kappa_{0}}{d \ln \mu}$

is the thermopower of the unmagnetized electron gas (cf. Urpin \& Yakovlev 1980b). The three terms in (2.15) are interpretable as:

(i) A convection of the field at a velocity given by the sum of the thermal diffusion velocity and the electron mobility. Note that at low field strength $(X \ll 1)$ the thermal drift is in the direction of the temperature gradient.

(ii) A battery term $\propto \vec{\nabla} n_{\mathrm{e}} \times \vec{\nabla} T$, which describes the creation of field by thermoelectric currents. If, as is usually the case, $\vec{\nabla} T \cdot \vec{B} \times \vec{F}>0$, this term will contain a part $\propto\left(-\vec{\nabla} n_{\mathrm{e}} \cdot \vec{F}\right) \vec{B}$ leading to exponential field growth when the heat flows down the density gradient. In a fluid the isotherms and equipotentials will coincide with the constant density surfaces and this term will vanish ${ }^{\star}$ ( $c f$. equation 2.13 ).

(iii) An ohmic decay term (third term in 2.15).

\section{Growth of magnetic field in the solid crust}

\subsection{STRUCTURE OF CRUST}

We are interested in the crust at densities high enough to solidfy at temperatures $T \sim 10^{8} \mathrm{~K}$ and low enough to be a poor enough electrical conductor to allow ohmic decay in a million years. These requirements confine our attention to the density range $10^{7} \mathrm{gm} / \mathrm{cm}^{3} \leqslant \rho \leqslant$ $10^{11} \mathrm{gm} / \mathrm{cm}^{3}$. Here the crust is supported by degenerate relativistic electron pressure. The mean molecular weight per electron, $\mu_{\mathrm{e}}$, and the nuclear charge, $Z$, vary as a function of density through the crust (e.g. Baym \& Pethick 1975); we adopt the average values $\mu_{\mathrm{e}}=$ 2.5 and $Z=32$ and use these throughout the crust. For a thin crust the depth below the surface is given as a function of density as

$z_{4}=1.5 \rho_{9}^{1 / 3} g_{14}^{-1}$

where $10^{4} z_{4} \mathrm{~cm}$ is the depth, $10^{9} \rho_{9} \mathrm{gm} / \mathrm{cm}^{3}$ is the density, and $10^{14} \mathrm{~g}_{14} \mathrm{~cm} / \mathrm{s}^{2}$ is the effective surface gravity. For a non-rotating star of gravitational mass $M$ and surface area $4 \pi R^{2}$ the

* A rather different view has been expressed by Dolginov \& Urpin (1980b) (cf. also Dolginov \& Urpin $1980 \mathrm{a})$. They consider the possibility of thermomagnetic instability within fluid white dwarfs. They perform a linearized perturbation analysis based on the induction and energy equations (2.15) and (2.1b). However, they do not include hydrostatic equilibrium, which should be achieved on time-scales far shorter than those associated with thermoelectric effects. In particular, in a fluid $\vec{\nabla} \rho \times \vec{\nabla} T=0$, which implies that the right-hand side of their equation $(7 \mathrm{~b})$ vanishes, along with their growth rate. 
surface gravity is

$g=\frac{G M}{R^{2}}\left(1-\frac{2 G M}{R}\right)^{-1 / 2}=\frac{1}{\rho} \frac{d P}{d z}$

Most models of $1.4 M_{\odot}$ neutron stars have radii in the range $8-16 \mathrm{~km}$ and surface gravities $0.85 \leqslant g_{14} \leqslant 4.2$.

The ions will form a Coulomb crystal when the plasma parameter $\Gamma\left[\Gamma \equiv(Z e)^{2} / a k_{\mathrm{B}} T\right.$, where $a$ is the interionic spacing] exceeds $\approx 150$ (Pollack \& Hansen 1973; Slattery, Doolen \& DeWitt 1980). Using the average values for $Z$ and $\mu_{\mathrm{e}}$ given above, the melting curve is

$T_{8}=3.6 \rho_{9}^{1 / 3}$.

Combining this with (3.1), we find that the ions will be crystalline below a depth

$z_{\mathrm{M}, 4}=0.4 T_{8} g_{14}^{-1}$

where $10^{8} T_{8} \mathrm{~K}$ is the temperature. At depths less than the melting depth, $z_{\mathrm{M}}$, the ions form a liquid metal.

\subsection{TRANSPORT COEFFICIENTS}

We require transport coefficients for both the solid and liquid metal regimes and consider two calculations: those of Flowers \& Itoh (1976) and Yakovlev \& Urpin (1980). We include electron-phonon scattering above the Debye temperature in the solid and electron-ion scattering in the liquid. Impurity scattering and the electron-electron contribution to the thermal conductivity, which is small for the large nuclear charges found in the crust, are neglected ( $c f$. Urpin \& Yakovlev 1980a). Quantizing effects of the magnetic field (Yakovlev 1980a,b; Kaminker \& Yakovlev 1980; Blandford \& Hernquist 1982) are neglected. Consider first the solid metal regime.

Electron-phonon scattering is elastic for temperatures well above the Debye temperature; the electron-phonon relaxation time is calculated explicitly by Yakovlev \& Urpin (1980). They obtain

$\tau_{\mathrm{YU}}=8.1 \times 10^{-19} T_{8}^{-1} \mathrm{~s}$

independent of density. The relaxation time may be extracted from table 3 of Flowers \& Itoh (1976) with the result that

$\tau_{\mathrm{FI}}=2.2 \times 10^{-18} T_{8}^{-1} \mathrm{~s}$

a factor of 2.7 larger than $\tau_{\mathrm{YU}}$. Note that (3.4) and (3.5) scale the same way with temperature and density. Yakovlev \& Urpin (1980) argue that the discrepancy between the calculations is due to their use of the Monte Carlo results of Pollack \& Hansen (1973) for the phonon spectrum of a Coulomb crystal, as opposed to Flowers \& Itoh's use of an approximate spectrum. In particular, Yakovlev \& Urpin (1980) use the Pollack \& Hansen (1973) value of the moment of the phonon spectrum $\left.u_{-2} \equiv<\omega^{-2}(k) / \omega_{\mathrm{p}}^{-2}\right\rangle=13$, where $\omega_{\mathrm{p}}$ is the ion plasma frequency. The approximation used by Flowers \& Itoh (1976) corresponds to $u_{-2}=4.4$. Flowers \& Itoh (Itoh 1982, private communication) argue that their calculation is more accurate due to their inclusion of electron screening $\left(k_{\mathrm{TF}}=1 / 4 q_{\mathrm{D}}\right.$ where $k_{\mathrm{TF}}$ is the Thomas-Fermi wavevector, and $q_{\mathrm{D}}$ is the Debye wavevector) and its neglect (Pollack \& Hansen assume a static neutralizing background of electrons) by Yakovlev \& Urpin (1980). 
We shall, without prejudice, use the Flowers \& Itoh (1976) value in our numerical estimates, and consider the relaxation time for electron-phonon scattering to be uncertain to a factor $\sim 3$. Using the relaxation time (3.5), the coefficients $\sigma_{0}$ and $\kappa_{0}$ are

$\sigma_{0}=1.8 \times 10^{22} \rho_{9}^{2 / 3} T_{8}^{-1} \mathrm{~s}^{-1}$

$\kappa_{0}=4.9 \times 10^{17} \rho_{9}^{2 / 3} \mathrm{erg} \mathrm{cm}^{-1} \mathrm{~s}^{-1} \mathrm{~K}^{-1}$.

Evaluating these at the melt surface, using (3.3a), gives

$\sigma_{\mathrm{M}}=1.4 \times 10^{21} T_{8} \mathrm{~s}^{-1}$

$\kappa_{\mathrm{M}}=3.8 \times 10^{16} T_{8}^{2} \mathrm{erg} \mathrm{cm}^{-1} \mathrm{~s}^{-1} \mathrm{~K}^{-1}$.

These coefficients must be reduced by a factor of 2.7 if the relaxation time (3.4) is used.

Transport coefficients in the liquid metal are, if anything, less certain than those in the solid. Comparison of the formulae for the thermal conductivity given by Yakovlev \& Urpin (1980) and Flowers \& Itoh (1981) yield a factor $\sim 2$ disagreement, with Flowers \& Itoh giving the larger value. The disagreement is due to different models for the ionic correlations and screening. Flowers \& Itoh (1976) screen the interaction using the Thomas-Fermi theory, and take the ionic correlations into account explicitly by modelling the results of Brush, Sahlin \& Teller (1966) to obtain a liquid structure function (see Flowers \& Itoh 1976 for details). Yakovlev \& Urpin (1980) ignore electron screening, which is a good approximation in the regime of interest $\left(k_{\mathrm{FT}} / p_{\mathrm{F}} \sim 0.1\right)$, and cut the interaction off at roughly the interionic distance. We shall again use the Flowers \& Itoh (1981) formula for the thermal conductivity due to electron-ion collisions which is a fit to the results of Flowers \& Itoh (1976) for the thermal conductivity of Baym, Pethick \& Sutherland (1971) matter. We omit the electronelectron scattering contribution to the thermal conductivity. This gives

$\kappa=10^{17} \rho_{9}^{1 / 3} T_{8} \operatorname{erg~cm}^{-1} \mathrm{~s}^{-1} \mathrm{~K}^{-1}$.

The electron-ion relaxation time implied by (3.10) is

$\tau=4.5 \times 10^{-19} \rho_{9}^{-1 / 3} \mathrm{~s}$.

\subsection{STATIONARY TEMPERATURE DISTRIBUTION WITH HORIZONTAL FIELD}

An important physical quantity in the problem is the ratio of the thermal diffusion time to the ohmic diffusion time. Numerically, this quantity is

$\frac{C_{\mathrm{v}}}{4 \pi \kappa_{0} \sigma_{0}}=5 \times 10^{-4}$

evaluated at the solid surface. The smallness of the ratio (3.12) means that the heat flow equilibrates rapidly, reaching a steady flow pattern in the presence of a slowly changing magnetic field. This behaviour is in contrast to that of laboratory metals; the ratio (3.12) for copper is $\sim 10^{3}$. Thermally driven growth of magnetic fields will not occur in laboratory solids since any magnetic field present will dissipate before significant temperature perturbations can be set up.

Due to the smallness of the ratio (3.12), which may be thought of as a magnetic Prandtl number, the temperature distribution may be calculated from the steady-state heat flow equation assuming a slowly changing magnetic field. In particular, consider a constant, 
vertical heat flux, $\vec{F}_{0}=-\kappa_{0} \vec{\nabla} T_{0}$, flowing through a plane-parallel, unmagnetized crust (the scale height of the crust is much less than the stellar radius so curvature effects may be ignored). Impose a small $(X \ll 1)$, magnetic field

$\vec{B}(x, z)=B(z) \sin (k x) \hat{y}$

and calculate the resulting temperature perturbation

$\delta T(x, z)=\delta T(z) \cos (k x)$.

Only the horizontal component of the magnetic field affects the heat flow for small fields, hence we have specialized to the case of a horizontal field.

The temperature distribution is given by the solution of the steady-state heat flow equation, which may be written ( $c f$. Landau \& Lifshitz 1960)

$\dot{E}=\vec{\nabla} \cdot(\vec{\kappa} \cdot \vec{\nabla} T)+\vec{j} \cdot(\overrightarrow{\vec{\sigma}})^{-1} \cdot \vec{j}-\overrightarrow{T j} \cdot(\vec{\nabla} \cdot \vec{Q})=0$

where $E$ is the internal energy density and a dot denotes a time derivative. If the temperature perturbation and the magnetic field are treated as small perturbations to the heat flow, (3.15a) may be simplified to

$\dot{E}=\vec{\nabla} \cdot(\vec{\kappa} \cdot \vec{\nabla} T)=0$

where the Joule heat term has been dropped because it is of second order in the magnetic field, and the Thomson effect term has been dropped because it is of order $(T / \mu)^{2}$ times the terms kept. The divergence of the electrical current has been set to zero. The divergence of the zeroth order heat flux is zero, so we are left with

$\vec{\nabla} \cdot \delta \vec{F}=0$

where the perturbation in the heat flux is given by

$\delta \vec{F}=-\kappa_{0} \vec{\nabla} \delta T-\frac{d \kappa_{0}}{d T} \delta T \vec{\nabla} T_{0}+\kappa_{0} \vec{X} \times \vec{\nabla} T_{0}$

The derivative $d \kappa_{0} / d T$ includes both the explicit temperature dependence of the thermal conductivity, which vanishes in our case, and the implicit temperature dependence due to the thermal expansion of the lattice. The thermal expansion effect may be shown to be negligibly small, thus we set the derivative $d \kappa_{0} / d T=0$. Setting to zero the divergence of (3.17), we obtain an equation for the temperature perturbation

$\frac{d}{d z}\left(\kappa_{0} \frac{d \delta T}{d z}\right)-\kappa_{0} k^{2} \delta T-k X\left|F_{0}\right|=0$

We now scale the depth in units of the melting depth, $z=z_{\mathrm{M}} \xi$, define $\beta=k z_{\mathrm{M}}$, use (3.1) and (3.7) to write $\kappa_{0}=\kappa_{\mathrm{M}} \xi^{2}$, and write $A=4 \pi e^{2} \kappa_{\mathrm{M}} \delta T / \mu_{\mathrm{M}}$ to obtain

$\frac{d}{d \xi}\left(\xi^{2} \frac{d A}{d \xi}\right)-\beta^{2} \xi^{2} A-\frac{\alpha \beta B}{\xi}=0$

where

$\alpha=\frac{4 \pi e^{2} \tau_{\mathrm{M}} z_{\mathrm{M}}\left|F_{0}\right|}{\mu^{2}}=\frac{\left|F_{0}\right| t_{\mathrm{M}}}{n_{\mathrm{M}} \mu_{\mathrm{M}} z_{\mathrm{M}}}$ 
is the diffusion velocity associated with the heat flux measured in units of the melting depth and the ohmic diffusion time-scale at the melt surface $t_{\mathrm{M}}=\left.4 \pi \sigma_{0} z^{2}\right|_{\mathrm{M}}$. In (3.20) $n_{\mathrm{M}}$ is the electron density at the melt surface. As is clear from (3.19), $\alpha$ describes the strength of the coupling between the magnetic field and the temperature perturbation.

Suitable boundary conditions for (3.19) are $\delta T=0$ at $z_{\mathrm{M}}$ and $\infty$. The former seems reasonable because the thermal time greatly exceeds the dynamical time in the liquid. The latter embodies our hypothesis that the field is confined to the surface layers. Equation (3.19) may be solved by a Green's function if the small variations of $T_{0}$ through the crust are neglected. We find

$A(\xi)=\frac{4 \pi e^{2} \kappa_{\mathrm{M}}}{\mu_{\mathrm{M}}} \delta T=\frac{\alpha \beta}{\xi} \int_{1}^{\infty} \frac{d \xi^{\prime}}{\xi^{\prime 2}} G\left(\xi, \xi^{\prime}\right) B\left(\xi^{\prime}\right)$

where $G\left(\xi, \xi^{\prime}\right)$ is given by

$\begin{array}{ll}G\left(\xi, \xi^{\prime}\right)=\sinh \beta(\xi-1) \exp [-\beta(\xi-1)] & 1 \leqslant \xi \leqslant \xi^{\prime} \\ G\left(\xi, \xi^{\prime}\right)=\sinh \beta\left(\xi^{\prime}-1\right) \exp [-\beta(\xi-1)] & \xi^{\prime} \leqslant \xi .\end{array}$

\subsection{LINEAR GROWTH OF THE MAGNETIC FIELD}

The evolution of the magnetic field is governed by the induction equation (2.15), with the temperature gradient computed from the heat flow equation (3.15a). For small fields the heat flow equation becomes (3.19). To linearize the induction equation write the field convection velocity, (2.16), as $\vec{V}=-\vec{F}_{0} / n_{\mathrm{e}} \mu$, where the relaxation time has been taken independent of density. The linearized induction equation is $\partial \vec{B} / \partial t=-\vec{\nabla} \times \delta \vec{\epsilon}$ where the perturbation in the electrochemical field is

$\delta \vec{\epsilon}=\vec{j} / \sigma_{0}+Q_{0} \vec{\nabla} \delta T+\vec{V} \times \vec{B}$.

The convection velocity is due to the perturbation of the thermopower caused by the magnetic field, $\delta \vec{Q} \cdot \vec{\nabla} T_{0}=\vec{V} \times \vec{B}$. If the perturbations (3.13) and (3.14) are used, along with the scalings leading to (3.19), the induction equation may be written in the form

$\frac{d}{d \xi}\left(\frac{1 d B}{\xi^{2} d \xi}\right)-\alpha \frac{d}{d \xi}\left(\frac{B}{\xi^{4}}\right)-\frac{\beta^{2} B}{\xi^{2}}+\frac{2 A}{\xi^{2}}=\lambda B$.

In deriving (3.24) the time dependence of the magnetic field has been assumed to have the form

$B(\xi, t)=B(\xi) \exp \left(\lambda t / t_{\mathrm{M}}\right)$

Equations (3.19) and (3.24) may be combined into a single fourth-order eigenvalue equation for the growth rate $\lambda$. Eliminating $A(\xi, t) \propto \delta T$ we find

$$
\begin{aligned}
& \frac{d^{4} B}{d \xi^{4}}-\frac{\alpha}{\xi^{2}} \frac{d^{3} B}{d \xi^{3}}+\left[\frac{6 \alpha}{\xi^{3}}-2 \beta^{2}\right] \frac{d^{2} B}{d \xi^{2}}+\left[\frac{\alpha \beta^{2}}{\xi^{2}}-\frac{18 \alpha}{\xi^{4}}\right] \frac{d B}{d \xi}+\left[\frac{24 \alpha}{\xi^{5}}+\beta^{4}-\frac{6 \alpha \beta^{2}}{\xi^{3}}\right] B \\
& =\lambda\left[\xi^{2} \frac{d^{2} B}{d \xi^{2}}+6 \xi \frac{d B}{d \xi}+\left(6-\beta^{2} \xi^{2}\right) B\right] .
\end{aligned}
$$


The fourth-order equation, (3.26), requires four boundary conditions. Two are provided by the requirement that the temperature perturbation vanish at the melt surface and at infinity. A third boundary condition is provided by the requirement that the magnetic field vanish at infinity. The fourth boundary condition is less certain. The rate of production of magnetic flux in the solid and the rate at which flux is convected in from the liquid may be calculated using Faraday's law and (3.23) for the perturbation of the electrochemical field. The magnetic field, (3.13), varies horizontally as $\sin (k x)$; to calculate the flux production and convection integrate Faraday's law over the surface $z \in\left[z_{\mathrm{M}}, \infty\right), x \in[0, \pi / k]$ and use Stokes' theorem to obtain, using (3.23) for $\delta \vec{\epsilon}$.

$\frac{d \Phi}{d t}=\int d \vec{l} \cdot\left(\vec{V} \times \vec{B}-Q_{0} \vec{\nabla} \delta T-\frac{\vec{\nabla} \times \vec{B}}{4 \pi \sigma_{0}}\right)$

where $\Phi=\int \vec{B} \cdot d \vec{\Sigma}$ is the magnetic flux. Specializing to the case $\vec{B}=B(\xi) \sin (k x) \hat{y}, \vec{V}=$ $V(\xi) \hat{z}$ and $\delta T=\delta T(\xi) \cos (k x)$ we see that the thermopower term $Q_{0} \vec{\nabla} \delta T$ is the only term which represents the production of flux. The convection and diffusion of flux through the melt surface is given by the integral of the $\vec{V} \times \vec{B}$ and the $(\vec{\nabla} \times \vec{B}) / 4 \pi \sigma_{0}$ terms along the melt. Evaluating these terms and transforming to the dimensionless variables used above, the rate of flux flow through the melt is found to be

$\left(\frac{d \Phi}{d t}\right)_{\mathrm{conv}}=\frac{2 z_{\mathrm{M}}^{2}}{\beta t_{\mathrm{M}}}\left[\alpha B-\frac{d B}{d \xi}\right]$

evaluated at the melt surface. Thus the condition $d B / d \xi=\alpha B$ represents all of the flux production taking place in the solid. In Section 4 the possibility of flux production in the liquid will be discussed, thus we shall discuss the eigenvalues $\lambda$ of (3.26) corresponding to various values of the logarithmic derivative $d \ln B / d \xi$.

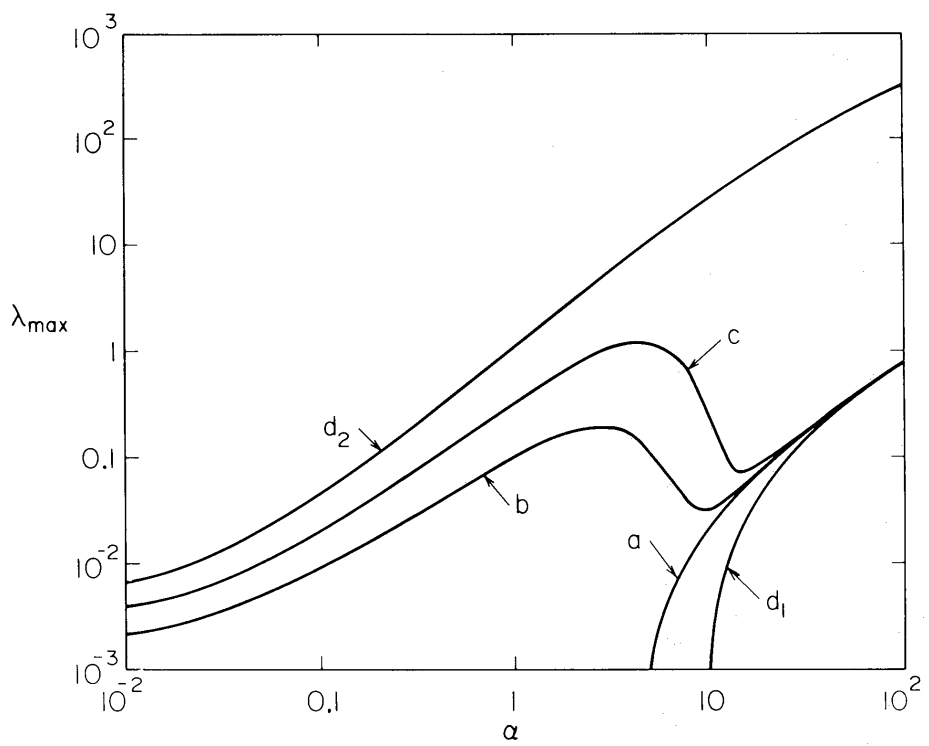

Figure 1. Maximum growth rate, $\lambda_{\max }$, with respect to $\beta$ as a function of $\alpha$ for various boundary conditions at $\xi=1$ : (a) $B^{\prime}=\alpha B$, (b) $B^{\prime}=0.75 \alpha B$, (c) $B^{\prime}=0.5 \alpha B$ and (d) $B^{\prime}=0$. For the boundary condition $B^{\prime}=0$ two different growing modes are present. Mode 1 resembles the growth mode for $B^{\prime}=\alpha B$ with all of the flux being produced in the solid. In mode 2 , all of the flux originates in the liquid and is convected across the solid surface into the solid crust. 


\subsection{NUMERICAL SOLUTIONS}

We have obtained numerical solutions to the fourth order eigenvalue equation (3.26) for a variety of boundary conditions. The solutions were obtained by writing (3.26) in finite difference form using the variable $s=e^{\xi}$. A grid of 75 equally spaced points on the interval $s \in[0,2.5]$ was employed. The boundary conditions at infinity were applied at the rightmost point of the grid. We consider two cases:

(a) The production of magnetic flux is confined to the solid. In this case the proper boundary condition is $d \ln B / d \xi=\alpha$ at the solid surface $\xi=1$. We find that a growing mode exists for $\alpha \geqslant 5$. In order for the growth rate to be large enough to be of interest in neutron star crusts $(\lambda \geqslant 0.1$, see Section 6) we require $\alpha \geqslant 22$ (Fig. 1). If we examine the eigenfunctions (Fig. 2) we find that the flux is concentrated well below the surface, near $\xi=3-4$.

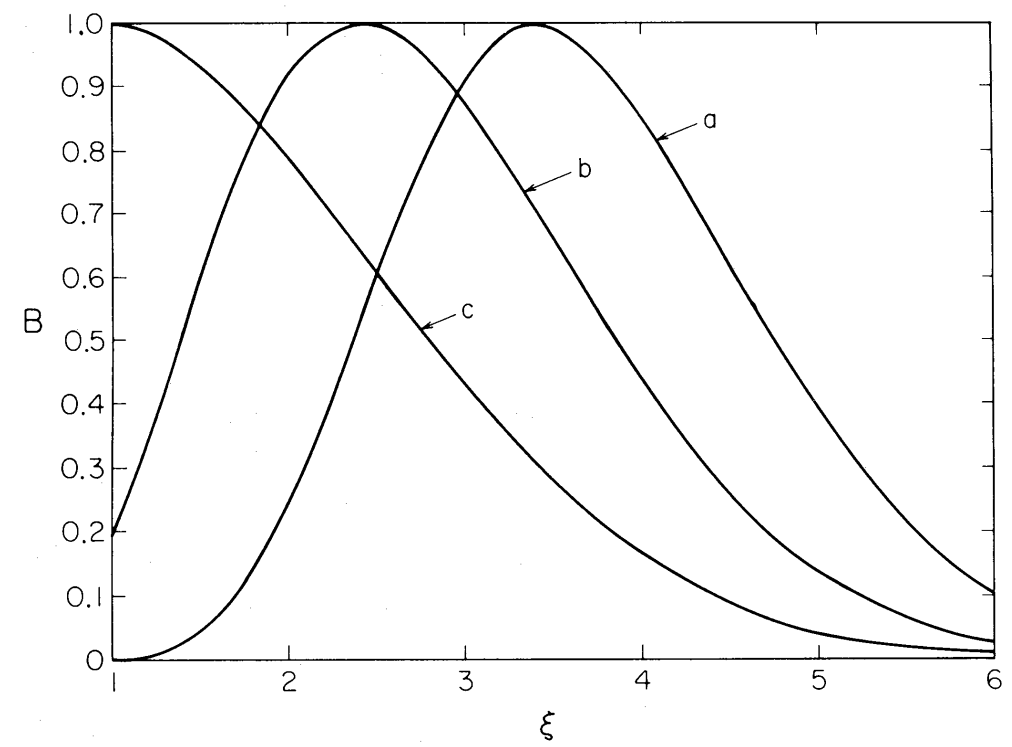

Figure 2. Eigenfunctions of growing modes with $\lambda_{\max }=0.1$ for three boundary conditions at $\xi=1$ : (a) $B^{\prime}=\alpha B$, (b) $B^{\prime}=0.75 \alpha B$ and (c) $B^{\prime}=0$. For $B^{\prime}=\alpha B$, all the flux is produced in the solid and $\alpha=22.5$. For $B^{\prime}=0.75 \alpha B 90$ per cent of the flux is produced in the liquid and $\alpha=5$. For $B^{\prime}=0$, (mode 2 in Fig. 1 ), all the flux originates in the liquid and $\alpha=0.2$.

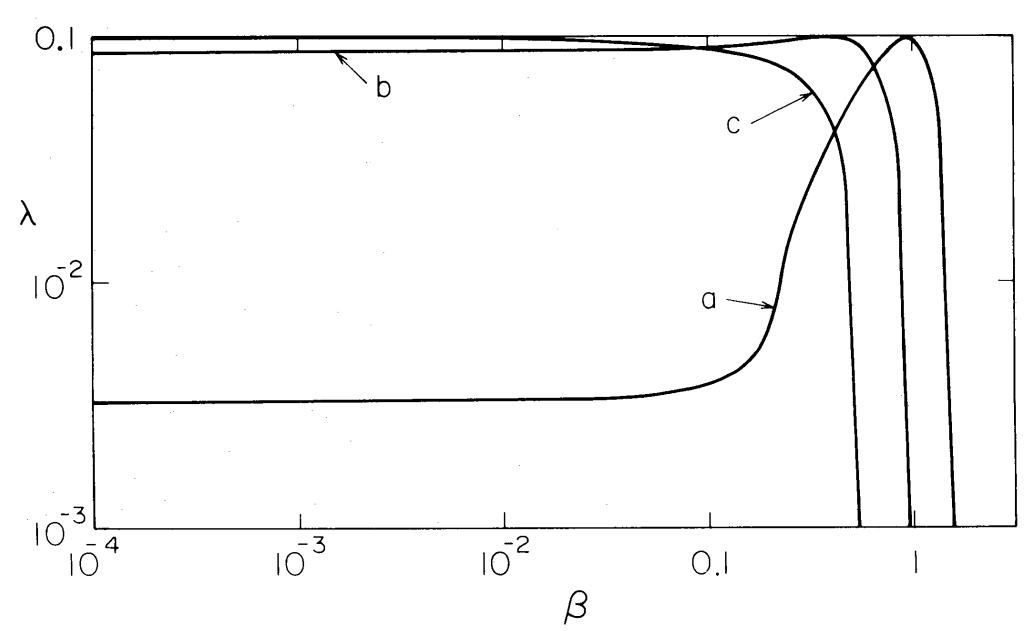

Figure 3. Growth rate as a function of $\beta$ for fixed $\alpha$ with $\lambda_{\max }=0.1$ for the boundary conditions (a) (c) of Fig. 2 . 
The explanation for this form of the eigenfunction can be seen from (3.24). For $1 \leqslant \xi \leqslant 5$ the convection term, (2.17), dominates ohmic loss and produces the steep vertical gradient of horizontal field, as $V \sim \xi^{-4}$. For $\xi \gtrsim 5$ ohmic loss dominates and the field strength decreases exponentially with depth. We find that the growth rates are maximized for $\beta \sim 1$ (Fig. 3 ). The horizontal and vertical field gradients are comparable, as expected.

(b) There is significant production of flux in the liquid. In this case flux will be convected into the solid crust from the liquid; the proper boundary condition at the melt surface is $d \ln B / d \xi<\alpha$. The amount by which the logarithmic derivative is lowered from the case in which all of the flux production takes place in the solid depends on the rate of flux production in the liquid. Efficient flux production in the liquid allows growing modes for values of $\alpha$ much lower than those needed in case (a). In particular, if we have $d \ln B / d \xi=$ $0.75 \alpha$ we obtain a growing mode with $\lambda \simeq 0.1$ for $1 \leqslant \alpha \leqslant 5$ (see Fig. 1 ). The fraction of the flux convected in from the liquid can be calculated using the eigenfunctions and the boundary condition $d \ln B / d \xi=0.75 \alpha$. If we consider the mode with $\alpha=5$, then we find that 90 per cent of the flux production takes place in the liquid. For the case $B^{\prime}=0$ a growing mode with $\lambda=0.1$ is obtained with $\alpha=0.2$ (see Figs 1 and 2).

\subsection{COMPARISON WITH COOLING CALCULATIONS}

A calculation of direct relevance to the subject of this paper has been performed by Gudmundsson et al. (1982) (see also Gudmundsson 1981). These authors have studied the variation of the heat flux through the outer crust assuming a fixed interior temperature, evaluated at $\rho \sim 10^{11} \mathrm{gm} / \mathrm{cm}^{3}$. They used electron conductive opacities from Yakovlev \& Urpin (1980) at high density, and the radiative and conductive opacities of the Los Alamos group (e.g. Hübner et al. 1977) at lower densities. They find an empirical relation, good to a few per cent, that relates the heat flux to the interior temperature and the surface gravity. Furthermore, they showed that the flux was almost completely controlled by the electron conduction opacity in the liquid at densities $10^{6} \mathrm{gm} / \mathrm{cm}^{3} \leqslant \rho \leqslant 10^{8} \mathrm{gm} / \mathrm{cm}^{3}$ for a central temperature $T=10^{8.5} \mathrm{~K}$. The controlling density range moves to lower density for lower central temperatures.

In common with earlier authors, Gudmundsson et al. confined their attention to a crust in local nuclear statistical equilibrium (i.e. composed of ${ }^{56} \mathrm{Fe}$ for densities $\rho<8 \times 10^{6} \mathrm{gm} /$ $\mathrm{cm}^{3}$, Baym et al. 1971). If we consider the response of the flux to changes in the composition of the crust in the controlling density range we find that the opacities scale as the nuclear charge, $Z$ and, for a given internal temperature, the flux scales as $1 / Z$, since the neutron star envelope tends strongly to the radiative zero solution (e.g. Schwarzschild 1958). The surface composition of neutron stars has been discussed by Michel (1975) and Rosen \& Cameron (1972) with the conclusion that $\sim 10^{21} \mathrm{gm}$ of helium could survive on the surface of the star. The helium will compress to a density given by $\Delta M=4 \pi R^{2} \int \rho d z=4 \times$ $10^{21} \rho_{6}^{5 / 3} g_{14}^{-1} \mathrm{gm}$. Thus a small amount of helium can reach a high enough density to be the controlling factor in determining the heat flux, lowering the effective $Z$ in (3.29) and (3.30) to $Z=2$ and substantially increasing the heat flux for a given internal temperature.

We have confirmed and generalized the Gudmundsson et al. relation, using the opacities given by Flowers \& Itoh (1981) and find, to adequate accuracy

$$
F \simeq 7 \times 10^{19} g_{14}\left(\frac{26}{Z}\right) T_{8}^{2.3} \mathrm{erg} \mathrm{cm}^{-2} \mathrm{~s}^{-1} \quad 0.3 \lesssim T_{8} \lesssim 3
$$


The flux is a factor $\sim 2$ larger than is obtained using the Yakovlev \& Urpin (1980) opacity. If we adopt this relation, then we find

$\alpha=0.5 T_{8}^{0.3}\left(\frac{26}{Z}\right)$

independent of the surface gravity, and only weakly dependent on the interior temperature.

The numerical value of $\alpha$ is sensitive to the collision time $\tau$. As can be seen from (3.20), $\alpha$ is proportional to the collision time at the melt surface. In addition, the heat flux $F_{0}$ is proportional to the collision time in the liquid (Gudmundsson et al. 1982). Thus, the factor of 2.7 discrepancy between the collision times of Flowers \& Itoh (1976) and Yakovlev \& Urpin (1980) leads to an overall factor $\sim 6$ uncertainty in the value of $\alpha$. This is in addition to the uncertainty in $\alpha$ due to ignorance of the surface composition.

\section{Growth of the field in the liquid phase}

As we showed in Section 2, the battery term which drives the linear growth of the field in the solid is absent in the liquid phase because hydrostatic equilibrium requires a vertical temperature gradient. However, this implies that a horizontal component of magnetic field will produce a horizontal component to the heat flux, given in the linear approximation by

$\delta \vec{F}=\vec{X} \times \vec{F}_{0}+O\left(X^{2}\right)$

where $\vec{F}_{0}=-\kappa_{0} \vec{\nabla} T_{0}$ is the vertical heat flux. For a general field geometry, this perturbation will not be solenoidal and as a result temperature perturbations will start to grow, thereby generating pressure perturbations and driving a circulation of the fluid in a manner akin to the Eddington-Sweet process (e.g. Schwarzschild 1958). The circulation velocity adjusts itself to convect away the local entropy production,

$T \rho(\vec{v} \cdot \vec{\nabla}) S+\vec{\nabla} \cdot \delta \vec{F}=0$

where $S$ is the entropy per unit mass and $\vec{v}$ is the circulation velocity. We rewrite equation (4.2) as

$$
\rho v_{\mathrm{z}}=-\frac{\vec{F} \cdot \vec{\nabla} \times \vec{X}}{T(d S / d z)}=\left[\vec{\nabla} \times\left(\frac{F_{0} \vec{X}}{T(d S / d z)}\right)\right]_{\mathrm{z}}
$$

where $F_{0}=\left|\vec{F}_{0}\right|$. Continuity of mass implies that $\vec{\nabla} \cdot \rho \vec{v}=0$; thus, a solution for the velocity field is

$\vec{v}=\frac{1}{\rho} \vec{\nabla} \times\left[\frac{F_{0} \vec{X}}{T(d S / d z)}\right]$.

This is the only solution that vanishes in the absence of a field. Equation (4.4) is valid for $X \ll 1$.

We are most interested in the circulation in the liquid close to the solidification point. Under these conditions the entropy is dominated by the ionic contribution, and the entropy gradient is dominated by the density gradient ( $d T / d z \ll T / z$ near the melting depth). We may use the results of Pollock \& Hansen (1973) to obtain

$\left(\frac{\partial S}{\partial n_{\mathrm{i}}}\right)_{\mathrm{T}}=-\frac{1.24}{\rho}$ 
where $n_{\mathbf{i}}$ is the density of ions. Equation (4.4) can then be rewritten

$\vec{v}=-\frac{e F_{0} \tau_{\mathrm{M}} A m_{\mathrm{p}}}{3.7 \rho_{\mathrm{M}} \mu_{\mathrm{M}} T} \frac{z_{\mathrm{M}}}{\xi^{4}} \vec{\nabla} \times(\vec{B} / \xi)$

where we have used the approximation

$\tau(\xi)=\tau_{\mathrm{M}} \xi^{-1}$

which is valid in the liquid $(\xi<1)$ (Yakovlev \& Urpin 1980).

There is a competing effect that dominates in normal stars (e.g. Parker 1979). Magnetic pressure contributes to the total pressure in the fluid and so, if there is to be static equilibrium, there must be associated temperature gradients. Individual magnetic filaments will rise or fall until they are at the same density as their surroundings and stable to buoyancy forces. The main difference in pressure between the inside and outside of a filament will be contributed by the ions, and so the temperature difference will satisfy $(\delta T / T) \sim\left(B^{2} / 8 \pi P_{\mathfrak{i}}\right)$ where $P_{\mathrm{i}}$ is the ion pressure. This will induce perturbations to the heat flux of magnitude $\delta F \sim\left(B^{2} / 8 \pi P_{\mathrm{i}}\right)(k z) F_{0}$, which are generally small compared to the perturbations caused by the anisotropy (4.1). Henceforth, we ignore this effect.

If the magnetic field is strong enough, it will be modified by the fluid motion faster than ohmic decay and the thermoelectric effects described in Section 2 can act. This requires that the circulation velocity be larger than the ohmic diffusion velocity and the field convection velocity. Fluid motion dominates if

$B \gtrsim(T / e \tau)[\min (1, \alpha)]^{-1}$

or

$B \gtrsim 10^{8} T_{8}^{2}[\min (1, \alpha)]^{-1}$.

When this inequality is strongly satisfied, the electric field will be given by

$\vec{E}=-\vec{v} \times \vec{B}=\frac{4 \pi K z_{\mathrm{M}}}{\xi^{4}} \vec{j} \times \vec{B}-\frac{K}{\xi^{6}}(\vec{B} \times \vec{\xi}) \times \vec{B}$

where

$K=\frac{e F_{0} \tau_{\mathrm{M}} A m_{\mathrm{p}}}{3.7 \rho_{\mathrm{M}} \mu_{\mathrm{M}} T}=5.5 \times 10^{-25} F_{19} T_{8}^{-6} G^{-1}$

where $F_{19}$ is the heat flux in units of $10^{19} \mathrm{erg} \mathrm{cm}^{-2} \mathrm{~s}^{-1}$. The magnetic field will then evolve according to $\partial \vec{B} / \partial t=-\vec{\nabla} \times \vec{E}$.

These thermal effects may drive a non-linear dynamo mechanism. The first term on the right-hand side of equation (4.10) dominates in the short wavelength limit, and describes the creation of horizontal field out of vertical field and vice versa - a crucial ingredient of a dynamo process. However, this term alone does not lead to enhancement of the magnetic energy density, as can be seen by noting that it does not contribute to the rate of performance of mechanical work, $-\vec{j} \cdot \vec{E}$. The second term on the right-hand side of (4.10) also represents the creation of perpendicular components of magnetic field, and can contribute to an increase in the magnetic energy density.

If thermally driven circulation in the liquid does drive a non-linear dynamo process the magnetic field in the liquid will grow to non-linear $(X \gg 1)$ strength in a time $t_{+} \sim z_{\mathrm{M}} / K B=$ $80 T_{8} / B_{8} g_{14} \mathrm{yr}$, shorter than the time-scale for field growth or decay in the solid. In this 
case most of the flux is made in the liquid and convected into the solid. The surface boundary condition in Section 3 will be determined by the rate of flux production in the liquid and the rate at which flux can be convected into the solid. This allows growing modes in the solid for values of $\alpha$ much smaller than those required if all of the flux production is to take place in the solid.

\section{Growth and decay of the magnetic field}

In the preceding two sections, we have described magnetic field generation in both the solid and the liquid under the assumption that the field is weak. Both calculations can break down for strong fields.

The magnetic stress in the liquid may become large enough to influence the circulation. The circulation velocity, given by equation (4.4), is sub-Alfvénic; hence, magnetic flux tubes will quickly adjust to hydromagnetic equilibrium. This implies that there will be small temperature perturbations on the equipotential surfaces of magnitude $\delta T / T \sim B^{2} / 8 \pi P_{\mathrm{i}}$ where $P_{\mathrm{i}}$ is the ion pressure, which is larger than the thermal component of the electron pressure. The circulation velocity (4.4) should be a reasonable approximation for magnetic field variations with $k z \sim 1$ as long as the perturbations to the heat flux $\sim \kappa_{0} \delta T / z$ associated with the temperature fluctuations are small compared to the perturbations due to the magnetic field $\sim X F_{0}$. The condition for this to be true is that

$B \lesssim 1.5 \times 10^{11} F_{19} g_{14}^{-1} \mathrm{G}$

or

$B \lesssim 10^{12} T_{8}^{2.3} \mathrm{G}$

assuming the relation (3.29), as we shall henceforth. An equivalent condition arises from the requirement that the magnetic stresses be smaller than the thermally induced pressure fluctuations and should therefore be unable to influence the circulation.

Secondly, the non-linear terms in equations (2.15) and (4.10) describing the evolution of the field in the solid and liquid respectively must be included when $X \gtrsim 1$ or

$B \gtrsim 2 \times 10^{11} T_{8}^{2} \mathrm{G}$.

Note that conditions (5.2) and (5.3) are similar for $T_{8} \sim 1$.

We expect that the end result of the evolution of the linear instability will be to produce a disorganized array of horizontal magnetic loops of size $\sim z$. However, when $X \gtrsim 1$, the horizontal temperature gradient should exceed the vertical temperature gradient. The dominant contributions to the convective velocity (2.16) are now

$\vec{V} \simeq-\frac{\vec{F}}{n_{\mathrm{e}} \mu}-\frac{\vec{j}}{n_{\mathrm{e}} e}$.

The battery term in (2.15) is unchanged. There is an additional field convection with the drift velocity of the conduction electrons. This term alone, like its counterpart in the fluid circulation analysis, causes no change in the total magnetic energy. However, when $X \gtrsim 1$, it dominates the ohmic decay term and leads to the production of vertical magnetic field out of horizontal field. The vertical field will penetrate the lower density regions where the thermal resistance is largest and where $X$, and therefore the inhibition of the cross-field thermal conductivity, is much larger. The vertical heat flux will therefore increase locally in those parts of the solid where the field is vertical, increasing the production rate of horizontal field. 
Horizontal electrical current and heat flux can also transport the field horizontally. Hence, magnetic field can be generated within one part of the stellar surface where the heat flux is large and then transported to another region where the field production is lower.

As a consequence of these effects, we believe that the rate of field growth in the solid is enhanced in the non-linear phase. What probably happens under conditions when field growth can occur is that magnetic flux is freely created in the vicinity of the solid surface and is then convected downwards into the crust with a speed $V \sim F / \mu n_{\mathrm{e}} \propto z^{-4}$. For $\xi \gg 1$ the ohmic diffusion term should dominate and in a time $10^{5} t_{5} \mathrm{yr}$, the flux should be able to diffuse down to a depth

$z_{\max } \sim 5 \times 10^{4} g_{14}^{-1 / 2} t_{5}^{1 / 4} T_{8}^{1 / 4} \mathrm{~cm}$.

As long as heat continues to flow through the crust, magnetic flux will be created and drawn downwards subject to two saturation effects. When the magnetic stress becomes comparable to the yield stress of the solid, the lattice will become visco-elastic and magnetic buoyancy will oppose field amplification. The shear modulus of the lattice is $4 x$ $10^{25} \rho_{9}^{4 / 3}$ dyne $\mathrm{cm}^{-1}$ (Ruderman 1972). If the lattice flows when the strain angle is $\sim 10^{-2}$, then the saturation field strength will be

$B_{\max } \sim 3 \times 10^{13} g_{14} \cdot t_{5}^{1 / 2} T_{8}^{-1 / 2} \mathrm{G}$

(Blandford \& Hernquist 1982).

Secondly, the temperature fluctuations induced by the magnetic field can no longer be regarded as perturbations when $X F / \kappa_{0} \gtrsim T / z$. This condition yields

$B_{\max } \gtrsim 10^{14} g_{14} T_{8}^{0.3} t_{5}^{0.5} \mathrm{G}$

independent of depth. The field can probably not grow to values much larger than this. This condition is similar to, but marginally weaker than, that given by the yield stress. (Note that the heat flux used in equation 5.7 may be reduced from the value appropriate to an unmagnetized crust. Note also that the field strength is not limited to the critical value $m_{\mathrm{e}}^{2} / e=4.4 \times 10^{13} \mathrm{G}$, Adler et al. 1970.)

After the magnetic field has reached its saturation value, it can still be moved around by the Hall currents. Adjacent loops of field will approach one another, establishing steep magnetic gradients which allow reconnection to proceed through $X$-type neutral points located near the surface where the resistivity is larger. We believe that there will be a steady progression towards larger scale magnetic structure until the horizontal scale length approaches the stellar radius. Thereafter, it seems that the only quasi-steady field geometry is one in which the field is axisymmetric and poloidal. In this case, the electrical current will be totally toroidal and the heat flux predominantly toroidal. If we estimate the integral

$\int_{0}^{\infty} B d z$

by $B_{\max } z_{\max }$ and assume that this is constant over the surface, then we can calculate the associated dipole moment. Flux conservation implies that the radial component of the field is $\propto \cot \vartheta$ where $\vartheta$ is the spherical polar angle. (This prescription clearly needs modification at the poles.) We can then expand in spherical harmonics to compute the magnetic dipole moment, $M$.

$M=\frac{3 \pi R^{2}}{8} \int B d z$

At this point we should comment upon the efficiency of the mechanisms that we have described. In the linear regime, a fraction $\sim B^{2} / 8 \pi \mu n_{\mathrm{e}} \sim B^{2} / 8 \pi \rho g z$ of the vertical heat flux is 
converted into magnetic energy density provided that ohmic losses are not dominant. As we have argued, this is probably a conservative estimate in the non-linear phase. However, this is always far below the maximum efficiency dictated by thermodynamics $\sim z \nabla T / T$ for a heat engine to do work against the magnetic stresses.

An axisymmetric magnetic structure can be maintained against ohmic loss as long as heat continues to flow through the crust. After the interior cools, the electrical conductivity will increase and the magnetic flux will diffuse away in a time dictated by its new value.

\section{Astrophysical applications}

Up to this point we have tried to determine general conditions under which magnetic field can grow in the outer crust of a neutron star. We now specialize by considering in turn slow pulsars, fast pulsars, pulsating X-ray sources, X-ray and $\gamma$-ray bursters, binary pulsars and white dwarfs.

\subsection{SLOW PULSARS}

The majority of radio pulsars appear to have been formed spinning slowly and to have dipole moments $\sim 10^{30} \mathrm{G} \mathrm{cm}^{3}$ which last $\sim 3 \times 10^{6} \mathrm{yr}$. Under the assumptions described in Section 3 we found that $\alpha \simeq 0.7$, independent of the surface gravity and weakly dependent on the temperature. Unless either the transport coefficients in both the liquid and the solid have been underestimated by factors $\sim 3$ or the surface layers are of helium composition, the linear instability described in Section 3 will not develop. However, if the neutron star retains a seed field $\gtrsim 10^{8} \mathrm{G}$, then, as discussed in Section 4 , magnetic flux can be created in the liquid and supplied to the solid.

The cooling of neutron stars has been a topic of considerable theoretical discussion since the Einstein X-ray satellite failed to discover hot neutron stars in most young supernova remnants. Cooling calculations have not agreed in detail with each other in part because of uncertainties in the transport properties of the matter in the crust. At early times the cooling is dominated by neutrino processes and the interior temperature is independent of the surface properties. For an unmagnetized $1.4 M_{\odot}$ neutron star with a superconducting interior and no pion condensate, (e.g. Richardson et al. 1982; Nomoto \& Tsuruta 1981) the interior temperature is roughly

$T_{8} \sim 1.3 t_{5}^{-0.2}$

where $t_{5} 10^{5} \mathrm{yr}$ is the age of the neutron star. This cooling law is valid until the photon flux from the surface $\sim 2 \times 10^{33} T_{8}^{2.2} \mathrm{erg} \mathrm{s}^{-1}$ for $M \sim 1.4 M_{\odot}$, is able to remove the internal energy $9 \times 10^{45} T_{8}^{2} \mathrm{erg}$. The cooling time is therefore

$t_{\text {cool }} \sim 2 \times 10^{5} \mathrm{yr}$.

This estimate of the cooling time will be increased if the magnetic inhibition of the heat flux in the liquid is taken into account.

If the growth rate, $\lambda \sim 0.1$ then a seed field of $10^{8} \mathrm{G}$ will growth to a strength $\sim 10^{12} \mathrm{G}$ in a time $t \sim 10 t_{\mathrm{M}} / \lambda \sim 10^{4} \mathrm{yr}$. This estimate is uncertain due to the dependence on the details of the non-linear evolution and the strength of the seed field; however, it does admit the possibility that most supernova remnants contain neutron stars in which the surface fields are sufficiently strong to reduce the surface X-ray luminosity but still sufficiently weak and disordered to have comparatively small dipole moments. (An investigation of the effects of magnetic fields on neutron star cooling is currently in progress.) In this way the theoretical expectation that type II supernova explosions generally produce neutron stars can be 
reconciled with the failure to observe them as either X-ray sources or radio pulsars. Recent searches for pulsars associated with supernova remnants in the Galaxy and the Magellanic Clouds, which avoid selection effects, have confirmed this discrepancy (Manchester, Tuohy \& D’Amico 1982.)

The field will growth to the limiting value, (5.6) or (5.7), in $\sim 10^{5} \mathrm{yr}$, and penetrate the crust to a depth $\sim 500 \mathrm{~m}$. If long range order has been established, the dipole moment will be $2 \times 10^{30} \mathrm{G} \mathrm{cm}^{3}$, using (5.8), in agreement with pulsar observations. Roughly $10^{-3}$ of the heat flux is converted into magnetic energy. No correlation between the magnetic and spin axes is expected, so most stars should be able to pulse.

After $\sim 2 \times 10^{5} \mathrm{yr}$, the interior of a pulsar should cool very rapidly. (The luminosity is usually larger than the rate of loss of spin energy.) A simple estimate gives $T_{8} \propto t_{5}^{-5}$. At a depth $\sim 500 \mathrm{~m}$, the Debye temperature is $\sim 4 \times 10^{8} \mathrm{~K}$. Well below the Debye temperature, the electron collision time and therefore the ohmic decay time increases unless limited by the presence of impurities (Urpin \& Yakovlev 1980b). In order for the decay time to be lengthened to the observed value of $\sim 3 \times 10^{8} \mathrm{yr}$, the conductivity must be increased by a factor $\sim 20$, which is certainly allowed but is by no means dictated by our theory.

\subsection{FAST PULSARS}

A subset of young pulsars, including those in the Crab, Vela and MSH 15-52 remnants are spinning comparatively rapidly and have inflated 'plerionic' nebulae around themselves. A large magnetic dipole moment seems to have formed very quickly in these objects. It is possible that the spin energy $\sim 10^{46} P^{-2} \mathrm{erg}$, is powering the production of subsurface field: perhaps through the dissipation of currents flowing through the magnetosphere. Another possibility is that additional heat flux is generated by friction between the core and the crust (Greenstein 1971).

The deceleration parameter, $n=-P \ddot{P} \mid \dot{P}^{2}+2$, has been measured for the Crab pulsar only with the result $n=2.5$, inconsistent with the simple electromagnetic pulsar theory (e.g Manchester \& Taylor 1977) which predicts $n=3$. The measured deceleration parameter can be reconciled with the simple theory if the field is still growing, as expected on the basis of our theory. Specifically, $B \propto t^{1 / 6}$ gives $n=2.5$.

The recent discovery of a $1.56 \mathrm{~ms}$ radio pulsar (Backer et al. 1982) for which $\dot{P}<10^{-15} \mathrm{~s}^{-1}$ (Backer 1982, private communication) is apparently inconsistent with this idea as the sur. face field strength is almost certainly $<10^{10} \mathrm{G}$. A possible explanation for this discrepancy is that the star is sufficiently massive to allow the formation of a central pion condensate (e.g Baym \& Pethick 1975). The core and inner crust would then cool very quicky by neutrinc emission. This would also be consistent with the absence of a detectable extended radic source surrounding the pulsar.

\subsection{PULSATING X-RAY SOURCES}

It is thought that the pulsating $\mathrm{X}$-ray sources in massive binary systems are older thar $\sim 10^{6} \mathrm{yr}$, and those systems with less massive primaries are even older, $\sim 10^{8} \mathrm{yr}$ in the case of Her X-1, since the binary does not become an X-ray source until the primary evolves off the main sequence (e.g. van den Heuvel 1977). Therefore, it is natural to postulate that the magnetic field in these stars is produced thermally as a result of accretion processes rathe than the initial cooling of the neutron star. As the X-ray luminosity of pulsating binary $\mathrm{X}$-ray sources is much greater than that of isolated, cooling neutron stars, the power tc generate magnetic fields is readily available. 
If the accreted gas were deposited at a uniform rate over the entire surface and the heat flow ere radially inward, then the battery term in (2.15) would lead to field destruction, and the field convection velocity, (2.16), would lead to the expulsion of field from the star (see the discussion following 2.15). However, it is far more probable that matter will be accreted over a small fraction of the surface and that heat will flow from the hot regions to the remainder of the surface via the interior since the thermal conductivity of the interior is much greater than that of the surface layers. We may idealize the problem as follows.

Consider a highly thermally conducting sphere of temperature $T_{2}$ and radius $R$ covered with a thin insulating layer. Let a fraction $f$ of the surface be maintained at a temperature $T_{1}$. Further assume that there exists a relationship $F(T)$ between the heat flux and the maximum temperature on either side of the layer. The central temperature will satisfy the relation $f F\left(T_{1}\right)=(1-f) F\left(T_{2}\right)$, as the heat conducted inward must balance the heat conducted outward for a steady state to exist. If we substitute equation (3.29) for $F(T)$, then we obtain

$T_{2} \sim\left[\frac{f}{(1-f)}\right]^{0.45} T_{1}$.

The time required to achieve equilibrium is roughly

$t_{\mathrm{eq}} \sim \frac{U}{4 \pi R^{2} f F\left(T_{1}\right)}$

where $U$ is the internal energy of the star.

For $L \sim 10^{38} \mathrm{erg} \mathrm{s}^{-1}$ and $f \sim 0.1$ the interior temperature is $\sim 10^{7} \mathrm{~K}$ and thermal equilibrium will be established after $\sim 3 \times 10^{5} \mathrm{yr}$. Using equation (5.8), the magnetic moment is $M \sim 4 \times 10^{29} t_{5}^{3 / 4}$. After $\sim 3 \times 10^{6} \mathrm{yr}$ the magnetic moment will be equal to the typically observed value $3 \times 10^{30} \mathrm{G} \mathrm{cm}^{3}$. Therefore, if a neutron star accretes at roughly the Eddington rate for a typical mass transfer time-scale (e.g. van den Heuvel 1976) it can generate its own magnetic field. Accretion at a much slower rate is probably not able to power magnetic field generation. This may have occurred in the majority of cases, in particular in the bulge sources with their low mass stellar companions (e.g. van den Heuvel 1977).

\subsection{X-RAY AND $\gamma$-RAY BURSTERS}

It has been proposed that $\mathrm{X}$ - and $\gamma$-ray bursters are differentiated by the absence or presence of a strong magnetic field (Woosley \& Wallace 1982). A neutron star is a binary system that has in the past experienced a large enough and long enough accretion rate will have generated a strong surface field and be susceptible to $\gamma$-ray bursting. A star that has accreted less gas will be essentially unmagnetized and observable as an X-ray burster.

\subsection{BINARY PULSARS}

Two of the three known binary pulsars (PSR $1913+16$ and $0655+64$ ) have anomalously small values of $\dot{P}$ (Damashek et al. 1982). Recently $\dot{P}$ was measured for the third binary pulsar PSR $0820+02$ (Manchester et al. 1982). Although the value obtained $(\dot{P}=0.125 \mathrm{x}$ $\left.10^{-15} \mathrm{~s}^{-1}\right)$ is not as small as for the other two binary pulsars, it is nevertheless significantly less than the $\dot{P}$ for most isolated radio pulsars. The inferred dipole moments are, therefore, small, $\sim 10^{29} \mathrm{G} \mathrm{cm}^{3}$ (somewhat larger for PSR $0820+02$, though still significantly smaller than values derived for isolated radio pulsars). It is also thought that these objects are older 
than the typical radio pulsars. In both cases it has been suggested that the observed pulsar was formed in a supernova explosion before the companion completed its evolution (Smarr \& Blandford 1976; Blandford \& DeCampli 1981; van den Heuvel 1981). It is possible that these neutron stars became magnetized during a very long X-ray binary phase and that the magnetic field penetrated to a greater depth than in an isolated pulsar, increasing the ohmic dissipation time.

\subsection{WHITE DWARFS}

Some white dwarfs are magnetized with surface fields $\lesssim 10^{8} \mathrm{G}$. However, none of the processes discussed in this paper are likely to be relevant for white dwarfs. Crystallization does not occur until white dwarfs are very old and little thermal energy remains. Thus, the processes described in Sections 2 and 3 cannot lead to efficient field production. In addition, the circulation velocity in the interior of a white dwarf (cf. Section 4) is only $V \sim F X / P_{\mathbf{i}} \sim$ $3 \times 10^{-9} B_{8} \mathrm{~cm} \mathrm{~s}^{-1}$. The turnover time therefore exceeds the cooling time for all reasonable field strengths.

\section{Conclusions}

In this paper we have endeavoured to present a complete and self-consistent description of neutron star magnetization that is an alternative to the usual view that the magnetic flux has been frozen into the collapsing core during the supernova explosion. We have argued on observational grounds that neutron star magnetic fields are ephemeral, and on theoretical grounds that the field must therefore be confined to the surface layers of the star.

We have extended the work of Urpin \& Yakovlev (1980b) and suggested two possible mechanisms for the generation of magnetic flux. We have shown how small seed fields can grow exponentially within the solid crust, and specified necessary conditions for this to occur. Our understanding of heat transport in neutron stars is still sufficiently uncertain, as evidenced by the difficulties posed by the X-ray observations of supernova remnants, that we do not know whether or not this instability can be responsible for the production of neutron star magnetic fields. Secondly, we have demonstrated that the coexistence of heat flux and magnetic field in the liquid will cause the fluid to circulate, which may lead to effective dynamo action. If so, seed fields in excess of $\sim 10^{8} \mathrm{G}$ will grow rapidly and supply flux to the solid below.

Our treatment of the subsequent evolution of the field is far more conjectural. We have sketched plausible mechanisms that may occur in the non-linear phase of field growth and lead to saturation, explaining the striking clustering of neutron star field strengths around $10^{12} \mathrm{G}$. Finally we have considered the consequences of the theory in the context of various types of neutron stars. The theory seems to account for the principal observed properties of neutron star magnetic fields.

The first priority for an improved understanding of these processes is a better description of the transport properties of the outer crust and the liquid above it. This should be possible in the near future. However, it appears that it will be far more difficult to solve for the non-linear and necessarily three-dimensional growth of the field in any satisfactory quantitative manner.

\section{Acknowledgments}

We thank the staff of NORDITA, where this work was initiated, for hospitality and especially Drs Gudmundsson and Pethick for guidance and encouragement. We thank Tom 
Hagstrom for assistance with the numerical computations. RB thanks the Director of the Institute for Theoretical Physics for hospitality during the completion of this paper and participants in the Space and Astrophysics Program for valuable comments. This research was supported by the National Science Foundation under grants AST80-17752 and PHY2727084 and the Alfred P. Sloan Foundation. JHA acknowledges the support of a Bantrell Fellowship at Caltech.

\section{References}

Adler, S. L., Bahcall, J. N., Callan, C. G. \& Rosenbluth, M. N., 1970. Phys. Rev. Lett., 25, 1061. Ashcroft, N. W. \& Mermin, N. D., 1976. Solid State Physics, Holt, Reinhart \& Winston, New York. Backer, D., Kulkarni, S., Heiles, C., Davies, M. \& Goss, W., 1982. IAU telegram.

Baym, G. \& Pethick, C., 1975. A. Rev. Nucl. Sci., 25, 27.

Baym, G., Pethick, C. \& Sutherland, P. G., 1971. Astrophys. J., 170, 299.

Biermann, L., 1950. Z. Naturf. A., 5, 65.

Blandford, R. D. \& DeCampli, W. M., 1981. In Pulsars, eds Sieber, W. \& Wielebinski, R., Reidel, Dordrecht, Holland.

Blandford, R. D., DeCampli, W. M. \& Königl, A., 1979. Bull. Am. Astr. Soc., 11, 703.

Blandford, R. D. \& Hernquist, L. E., 1982. Journ. Phys. C., 15, 6233.

Brush, S. G., Sahlin, H. L. \& Teller, E., 1966. J. Chem. Phys., 45, 2102.

Damashek, M., Backus, P. R., Taylor, J. H. \& Burkhardt, R. K., 1982. Astrophys. J., 253, L57.

Dolginov, A. Z. \& Urpin, V. A., 1980a. Sov. Astr., 24, 177.

Dolginov, A. Z. \& Urpin, V. A., 1980b. Astrophys. Space Sci., 69, 259.

Fenimore, E. E., Laros, J. G., Klebesadel, R. W., Stockdale, R. E. \& Kane, S., 1982. In Gamma Ray Transients and Related Astrophysical Phenomena, eds. Lingenfelter, R. E., Hudson, H. S. \& Worrall, D. M. American Institute of Physics, New York.

Flowers, E. \& Ruderman, M. A., 1977. Astrophys. J., 215, 302.

Flowers, E. \& Itoh, N., 1976. Astrophys. J., 206, 218.

Flowers, E. \& Itoh, N., 1981. Astrophys. J., 250, 750.

Ghosh, P. \& Lamb, F. K., 1979. Astrophys, J., 234, 296.

Glen, G. \& Sutherland, P. G., 1980. Astrophys. J., 239, 671.

Goldreich, P., 1970. Astrophys. J., 160, L11.

Greenstein, G. 1971. Nature, 232, 117.

Gudmundsson, E. H., 1981. Unpublished Thesis, University of Copenhagen.

Gudmundsson, E. H., Pethick, C. \& Epstein, R. I., 1982. Astrophys. J., 259, L19.

Gunn, J. E. \& Ostriker, J. P., 1970. Astrophys. J., 160, 979.

Helfand, D. J., Chanan, G. A. \& Novick, R., 1980. Nature, 283, 337.

Hibberd, F. H., 1979. Proc. R. Soc. Lond., A369, 31.

Hübner, W. F., Merts, A. L., Magee, N. H., Jr \& Argo, M. F., 1977. Astrophysical Opacity Table, Report No. LA6760M, Los Alamos.

Inoue, H., Koyama, K., Makishima, K., Matsuoka, M., Murakomi, T., Oda, M., Ogarawa, Y., Ohashi, T., Shibazaki, N., Tanaka, Y., Kondo, I., Hayakawa, S., Kunieda, H., Makino, F., Masai, K., Nagase, F., Tawara, I., Miyamoto, S., Tsunemi, H. \& Yamashita, K., 1981. Astrophys. J., 250 , L71.

Joss, P. C., 1980. A. Rev. New York Acad. Sci., 336, 479.

Kaminker, A. D. \& Yakovlev, D. G., 1980. Preprint No. 681 A. F. Ioffe Institute of Physics and Technology, Leningrad.

Landau, L. D. \& Lifshitz, E. M., 1960. Electrodynamics of Continuous Media, Pergamon Press, Oxford.

Lyne, A. G., 1981. In Pulsars, eds Sieber, W. \& Wielebinski, R., Reidel, Dordrecht, Holland.

Manchanda, R. K., Bazzano, A., La Padula, C. D., Polcaro, V. F. \& Ubertini, P., 1982. Astrophys, J., $252,172$.

Manchester, R. N., 1981. In Pulsars, eds Sieber, W. \& Wielebinski, R., Reidel, Dordrecht, Holland.

Manchester, R. N., Newton, L. M., Cooke, D. J., Backus, P. R., Damashek, M., Taylor, J. H. \& Condor, J. J., 1982. Preprint.

Manchester, R. N. \& Taylor, J. H., 1977. Pulsars, Freeman and Co., San Francisco.

Manchester, R. N., Tuohy, J. R. \& D'Amico, N., 1982. Preprint.

Maurer, G. S., Johnson, W. N., Kurfess, J. D. \& Strickman, M. S., 1982. Astrophys. J., 254, 271. 
Mazets, E. P., Golenetskii, S. V., Apetekar', R. L., Gur'yan, Yu. A. \& Ill'inskii, V. N., 1981. Nature, $290,378$.

Mestel, L., 1961. Mon. Not. R. astr. Soc., 122, 473.

Michel, F. C., 1975. Astrophys. J., 198, 683.

Nomoto, K. \& Tsuruta, S., 1981. Astrophys. J., 250, L19.

Parker, E. N., 1979. Cosmical Magnetic Fields, Clarendon Press, Oxford.

Pollock, E. L. \& Hansen, J. P., 1973. Phys. Rev. A., 8, 3110.

Pravdo, S. H., White, N. E., Boldt, E. A., Holt, S. S., Serlemitsos, P. J., Swank, J. H., Szymkowiak, A. E., Tuohy, I. \& Garmire, G., 1979. Astrophys. J., 231, 912.

Rappaport, S. \& Joss, P. C., 1977. Nature, 266, 683.

Richardson, M. B., Van Horn, H. M., Ratcliff, K. F. \& Malone, R. C., 1982. Astrophys. J., $255,624$.

Rosen, L. C. \& Cameron, A. G. W., 1972. Astrophys. Space Sci., 15, 137.

Roxburgh, I. W., 1966. Mon. Not. R. astr. Soc., 132, 201.

Ruderman, M. A., 1972. A. Rev. astr. Astrophys., 10, 427.

Ruderman, M. A. \& Sutherland, P. G., 1973. Nature Phys. Sci., 246, 93.

Schwarzschild, M., 1958. Structure and Evolution of the Stars, Dover Publications, New York.

Seward, F. D. \& Harnden, F. R. Jr, 1982. Astrophys. J., 256, L45.

Seward, F. D., Harnden, F. R. Jr, Murdin, P. \& Clark, D. H., 1982, Preprint.

Slattery, W. L., Doolen, G. D. \& DeWitt, H. E., 1980. Phys. Rev. A, 21, 2087.

Smarr, L. L. \& Blandford, R. D., 1976, Astrophys, J., 207, 574.

Stamper, J. A., Papadopoulos, K., Sudan, R. N., Dean, S. O., McLean, E. A. \& Dawson, J. M., 1971. Phys. Rev. Lett, 26, 1012.

Trümper, J., Pietsch, W., Reppin, C., Voges, W., Staubest, R. \& Kendziorra, E., 1978. Astrophys. J., 219, L105.

Tsuruta, S., 1979. Phys. Rept., 56, 237.

Tuohy, I. \& Garmire, G., 1980. Astrophys. J., 239, L107.

Urpin, V. A. \& Yakovlev, D. G. 1980a. Soviet Astr., 24, 126.

Urpin, V. A. \& Yakovlev, D. G. 1980b. Soviet Astr., 24, 425.

'van den Heuvel, E. P. J., 1976. In Structure and Evolution of Close Binary Systems, eds Eggleton, P.et al., Reidel, Dordrecht, Holland.

van den Heuvel, E. P. J., 1977. A. N.Y. Acad. Sci., 302, 14.

van den Heuvel, E. P. J., 1981. In Pulsars, eds Sieber, W. \& Wielebinski, R., Reidel, Dordrecht, Holland.

Van Riper, K. A. \& Lamb, D. Q., 1981. Astrophys. J., 244, L13.

Vivekanand, M. \& Narayan, R., 1981. Astrophys. Astr., 2, 315.

Vivekanand, M. \& Radhakrishnan, V., 1981. In Pulsars, eds Sieber, W. \& Wielebinski, R., Reidel, Dordrecht, Holland.

Weisskopf, M. C., Elsner, R. F., Darbro, W., Leahy, D., Naranan, S., Sutherland, P. G., Grindlay, J. E., Harnden, F. R. Jr \& Seward, F. D., 1982. Astrophys. J., in press.

Wheaton, Wm. A., Doty, J. P., Primini, F. A., Cooke, B. A., Dobson, C. A., Goldman, A., Hecht, M., Hoffman, J. A., Howe, S. K., Scheepmaker, A., Tsiang, E. Y., Lewin, W. H. G., Matteson, J. L., Gruber, D. E., Baity, W. A., Rothschild, R., Knight, F. K., Nolan, P. \& Peterson, L. E., 1979. Nature, 282, 240.

Woosley, S. E. \& Wallace, R. K., 1982. Preprint.

Yakovlev, D. G., 1980a. Preprint No. 678, A. F. Ioffe Institute of Physics and Technology, Leningrad. Yakovlev, D. G., 1980b. Preprint No. 679, A. F. Ioffe Institute of Physics and Technology, Leningrad.

Yakovlev, D. G. \& Urpin, V. A., 1980. Soviet Astr., 24, 303.

Ziman, J. M., 1972. Principles of the Theory of Solids, Cambridge University Press. 